\title{
Detection of human cytomegalovirus in normal and neoplastic breast epithelium
}

\author{
Lualhati E Harkins', Lisa A Matlaf², Liliana Soroceanu², Katrin Klemm¹, William J Britt ${ }^{3}$, Wenquan Wang ${ }^{4}$, \\ Kirby I Bland ${ }^{5}$, Charles S Cobbs ${ }^{2 *}$
}

\begin{abstract}
Introduction: Human cytomegalovirus (HCMV) establishes a persistent life-long infection, and can cause severe pathology in the fetus and the immunocompromised host[1]. Breast milk is the primary route of transmission in humans worldwide, and breast epithelium is thus a likely site of persistent infection and/or reactivation, though this phenomenon has not previously been demonstrated. Increasing evidence indicates HCMV infection can modulate signaling pathways associated with oncogenesis. We hypothesized that persistent HCMV infection occurs in normal adult breast epithelium and that persistent viral expression might be associated with normal and neoplastic ductal epithelium.
\end{abstract}

Methods: Surgical biopsy specimens of normal breast $(n=38)$ breast carcinoma $(n=39)$ and paired normal breast from breast cancer patients $(n=21)$ were obtained. Specimens were evaluated by immunohistochemistry, in situ hybridization, PCR and DNA sequencing for evidence of HCMV antigens and nucleic acids.

Results: We detected HCMV expression specifically in glandular epithelium in 17/27 (63\%) of normal adult breast cases evaluated. In contrast, HCMV expression was evident in the neoplastic epithelium of 31/32 (97\%) patients with ductal carcinoma in situ (DCIS) and infiltrating ductal carcinoma (IDC) cases evaluated ( $p=0.0009$ ).

Conclusions: These findings are the first to demonstrate that persistent HCMV infection occurs in breast epithelium in a significant percentage of normal adult females. HCMV expression was also evident in neoplastic breast epithelium in a high percentage of normal and neoplastic breast tissues obtained from breast cancer patients, raising the possibility that viral infection may be involved in the neoplastic process.

\section{Introduction}

Environmental and epidemiological factors that contribute to breast cancer are poorly understood, and only $5-20 \%$ of women with breast cancer are known to have hereditary risk factors [2]. While investigators have searched for viruses that contribute to breast cancer pathogenesis, no causal associations have been established [3]. An association of Epstein-Barr virus (EBV) with breast cancer has been reported in the literature [4-7]. These reports have principally relied upon use of the DNA detection techniques of PCR and Southern blot hybridization, but these data have not been validated with appropriately sensitive in situ techniques. Other investigators have found that human endogenous

\footnotetext{
* Correspondence: charles.cobbs@gmail.com

${ }^{2}$ Research Institute, California Pacific Medical Center, (475 Brannan St., Suite 220) San Francisco CA, 94107, USA

Full list of author information is available at the end of the article
}

retroviruses with homology to mammary tumor virus are associated with a significant percentage of breast cancer, and can contribute to epithelial cell transformation both in vitro and in vivo[8-13]. Human papilloma virus (HPV) is a known oncogenic virus that has been detected in breast cancer cell lines and breast tumor cells[14-17]. The viral proteins E6 and E7 are capable of immortalizing normal human breast epithelial cells, however it is unclear whether HPV has a causal role in breast cancer[18].

Increasing evidence in the last 10 years suggests that human cytomegalovirus (HCMV) is associated with several human malignancies, including malignant glioma, colorectal carcinoma, prostate cancer, and skin cancer, and that HCMV gene products can modulate oncogenic properties of cells in vitro [19-26]. HCMV gene products can dysregulate cell cycle progression, cause DNA mutations, block apoptotic pathways,
C Biomed Central

(c) 2010 Harkins et al; licensee BioMed Central Ltd. This is an Open Access article distributed under the terms of the Creative Commons Attribution License (http://creativecommons.org/licenses/by/2.0), which permits unrestricted use, distribution, and reproduction in any medium, provided the original work is properly cited. 
inhibit immune response, and inhibit tumor suppressor protein functions [27-33]. Thus, cells that are persistently or abortively infected with HCMV might be at increased risk of developing genomic instability and immunological privilege, which could accelerate neoplastic transformation.

While evidence of HCMV in human breast glandular tissues is lacking, breast glandular epithelium is a likely reservoir for persistent HCMV infection in humans. Breast milk represents an established primary route of HCMV transmission in humans, and the shedding of cell free virus occurs in the breast milk of over $90 \%$ of women who are seropositive for HCMV [34-38]. Since persistent HCMV infection of breast epithelium could, in theory, promote malignant transformation of infected breast epithelium, we sought to determine the detection of HCMV gene products in normal and neoplastic breast. To this end, we used highly sensitive immunohistochemical (IHC) and in situ hybridization techniques (ISH) to analyze archived paraffin-embedded non-neoplastic breast tissues (from reduction mammoplasty patients) and breast carcinoma specimens with matched non-neoplastic appearing breast tissues for evidence of HCMV antigens and nucleic acids. This report is the first to demonstrate that persistent HCMV infection occurs in a significant percentage of non-neoplastic breast tissues. Moreover, we find that HCMV infects a very high percentage of both non-neoplastic and neoplastic breast epithelium from patients with breast cancer.

\section{Materials and methods Clinical Samples}

Formalin-fixed, paraffin-embedded, surgical biopsy specimens were obtained from histopathologically normal breast tissues (from patients undergoing elective breast reduction mammoplasty with no known history of breast cancer), neoplastic and non-neoplastic adjacent breast tissues from breast cancer patients from the pathology tissue procurement archives of the University of Alabama at Birmingham and the Birmingham Veterans Affairs Hospital. The ages of breast cancer patients ranged from 22-80, (mean = 48). Ages ranged from 2057 in the normal breast (reduction mammoplasty) group (mean $=36)$. All samples were obtained in accordance with ethics guidelines from the institutional review board of each institution. A faculty pathologist (K. K.) re-examined all cases to confirm histological diagnosis. Since these paraffin embedded specimens were part of the patient record, we were not allowed to exhaust the specimen. Some specimens had only limited amounts of tissue available, and we were therefore not able to perform all of the immunohistochemical and in situ hybridization studies on each specimen. For this reason the total number of specimens used exceeded the number analyzed for any given reagent.

\section{Immunohistochemical analyses of paraffin sections}

4- $\mu \mathrm{m}$ paraffin sections were procured from biopsy specimens of neoplastic and non-neoplastic breast, deparaffinized in xylene, and hydrated them in graded alcohols. Samples were processed as previously described [25] using monoclonal antibodies for immunodetection (antiIE1/2 ["IE"; IgG 1 isotype, 1:40, Chemicon, Temecula, $\mathrm{CA}$, anti-CMV cocktail, containing antibodies specific for early and late antigens ["E/L"; $\operatorname{IgG}_{2 \alpha}$ isotype,1:40, Innovex Biosciences, Richmond, $\mathrm{CA}]$, and anti-CMV late antigen ["L"; $\operatorname{IgG}_{2 \alpha}$ isotype, 1:40, Chemicon, Temecula, CA]). As controls we used anti-CD34 (1:40, BioGenex), and anti-smooth muscle actin (1:40; BioGenex, San Ramon, CA), and omission of primary antibody (no antibody). Immunostaining with the different antibodies was performed in a blinded fashion with respect to tissue diagnosis. One pathologist (K. K.) who was blinded to the antibody used analyzed immunostaining results and sections were called positive if specific immunoreactivity was detected.

\section{In situ hybridization of paraffin sections}

To confirm that HCMV nucleic acids were present in the pathological sections, the investigators performed in situ hybridization with a commercially available HCMV oligonucleotide cocktail probe labeled with fluorescein (BioGenex/Innogenex, San Ramon, CA). This probe consisted of six fluorescein isothiocyanate (FITC)-conjugated 40-mer probes spanning coding regions within the HCMV IE1 gene, and did not hybridize with human DNA sequences. Positive control probe specific for human Alu DNA sequences and negative control probe specific to an insect virus genome, both provided by the manufacturer, were also used. 4- $\mu \mathrm{m}$ paraffin sections were cut, deparaffinized, and hydrated through an established series of graded ethanol. Status of fixation was assessed for all cases before proceeding, and sections were post-fixed in formalin if necessary. After treatment, the prepared slides were rinsed in distilled water, dehydrated to $100 \%$ ethanol and air dried. Prediluted probe was then placed onto the sections, a cover slip was applied and slides were denatured on a MISHA thermocycler (Shandon Lipshaw/Hybaid Omnigene) at $90^{\circ} \mathrm{C}$ for 8-10 minutes, and then hybridized at $37^{\circ} \mathrm{C}$ in humidified chamber overnight. Slides were washed in TBST buffer, subjected to probe wash $(0.05 \%$ SSC buffer $20 \mathrm{~min}$. at $40^{\circ} \mathrm{C}$ ), then washed in $1 \times$ PBS. Endogenous avidin, biotin and Fc receptors were then blocked using avidin/ biotin blocking kit (BioGenex, San Ramon, CA) and Fc block (Innovex Biosciences, Richmond, CA). Fluoresceinlabeled probe was then detected using Supersensitive ${ }^{\circledR}$ in 
situ detection system (Innogenex, San Ramon, CA) with the chromogen BCIP/NBT.

\section{PCR and DNA sequencing}

DNA was purified from paraffin sections (3-6 $10 \mu \mathrm{m} \mathrm{sec-}$ tions) cut from a subset of the same biopsy specimens described above using DNeasy Tissue System (Qiagen, Valencia, CA) per manufacturer's instructions. To avoid potential PCR contamination, these experiments were performed in a laboratory devoid of previous exposure to infectious or recombinant HCMV. All preparations were processed throughout in a blinded fashion; no positive controls were used in any PCR reactions and blank paraffin blocks were cut sequentially between each patient sample and processed identically. For preparation of each case, the sectioning blade was replaced and the cutting surface was cleansed with xylene and ethanol. From each sample, 100-250 ng of DNA was amplified by nested PCR using internal and external primers specific for HCMV glycoprotein B (UL55) gene as described [39]. Samples were considered positive when a band of $140 \mathrm{bp}$ size could be visualized on agarose gel with ethidium bromide. Amplified DNA products were visualized on $1.5 \%$ agarose gels with ethidium bromide, bands were cut out, and DNA was extracted (gel extraction kit, Qiagen, Valencia, CA) and analyzed by automated sequencing (ABI Model 377 DNA Sequencer, Foster City, CA). Confirmation of HCMV sequence was performed using a NCBI Blast search.

\section{Statistical analyses}

Immunohistochemical data were determined in breast tumor samples, breast tumor control samples, and normal control samples. The percentages of positive sections for each specific monoclonal antibody in each group of samples were estimated. Chi-square test or Fisher's exact test was applied to compare tumor samples or tumor controls samples to normal control samples.

\section{Results}

\section{Immunohistochemistry for HCMV}

To determine whether HCMV was present in breast epithelium from normal controls and breast cancer patients, archived formalin fixed paraffin embedded breast tissues were utilized. Non-neoplastic breast tissues from breast reduction mammoplasty patients was used as "non-neoplastic normal" control $(\mathrm{N}=38)$. To evaluate HCMV presence in neoplastic tissues, the investigators obtained tumor biopsy specimens $(\mathrm{N}=39)$ and histopathologically non-neoplastic biopsy specimens from many of the same patients ("tumor control", $\mathrm{N}=21$ ).

To detect HCMV protein expression, we performed immunohistochemistry on as many specimens as possible with a panel of monoclonal antibodies that were specific to HCMV antigens expressed at different stages of the viral life cycle. Due to limitations on the use of archived patient specimens and/or tissue quality, not all specimens could be evaluated for all antibodies and in situ hybridization. The antibodies used are specific for HCMV immediate early ("IE"), early and late ("E/ L"), or late antigens ("L") (Figures 1 and 2). As controls, we used monoclonal antibodies specific for smooth muscle actin (SMA) (data not shown) and CD34 (Figure $1,1)$. These antibodies react to smooth muscle cells and vascular endothelial cells, respectively, but not breast epithelial cells, and serve as $\operatorname{IgG}_{2 \alpha}$ and $\operatorname{IgG}_{1}$ isotype positive and negative monoclonal antibody controls, respectively. As additional negative controls, we performed immunostaining in the absence of primary antibody for all cases (data not shown).

We detected HCMV-IE antigens in neoplastic epithelium from $97 \%$ of breast cancers tested while IE antigens were detected in only $63 \%(p=0.0009)$ of normal control cases tested (Table 1, Figure 1). IE immunoreactivity occurred in normal appearing ductal epithelium (Figure 1, a-b), epithelium in DCIS, and in IDC epithelial cells (Figure 2, a) evident in tumor specimens, but IE immunoreactivity was not observed in stromal cells (Figure 1, a-f). Immunoreactivity with the HCMV-E/L monoclonal antibody cocktail was detected in $84 \%$ of breast cancer specimens tested; only $21 \%$ of normal breast controls had confirmed immunoreactivity ( $\mathrm{p}<$ 0.0001; Table 1). The HCMV-E/L immunoreactivity was detected in a similar cellular pattern as that of IE antigen, with a staining pattern restricted to epithelium (Figure 1, g-h; Figure 2, b). Monoclonal antibody immunoreactivity with HCMV-L was detected in $56 \%$ of breast cancer specimens and 39\% of normal controls $(p=0.227$; Table 1). The pattern of late antigen cellular localization was also similar to IE and E/L immunoreactivity (Figure 1, j, k; Figure 2, c). HCMV late antigen was detected, in general, at a less intense level, although occasional rare cells were very intensely positive for late antigen (Figure 2, c). In some of the tumors there were areas of homogenous low level immunoreactivity to IE, $\mathrm{E} / \mathrm{L}$ and $\mathrm{L}$ antigens, while in other tumors there were scattered foci of positive tumor cells. The intensity of immunostaining varied significantly from tumor cell to tumor cell within any given tumor (e.g., Figure 1, j, k; Figure 2).

In general, we detected little HCMV immunohistochemical staining of stromal fibroblasts in the tumor cases. We did, however, perform immunostaining on a subset of tumor cases with a monoclonal antibody specific to the HCMV pp65 tegument antigen. In several cases we detected intense immunostaining of infiltrating stromal macrophages with this antibody (see Additional file 1). 

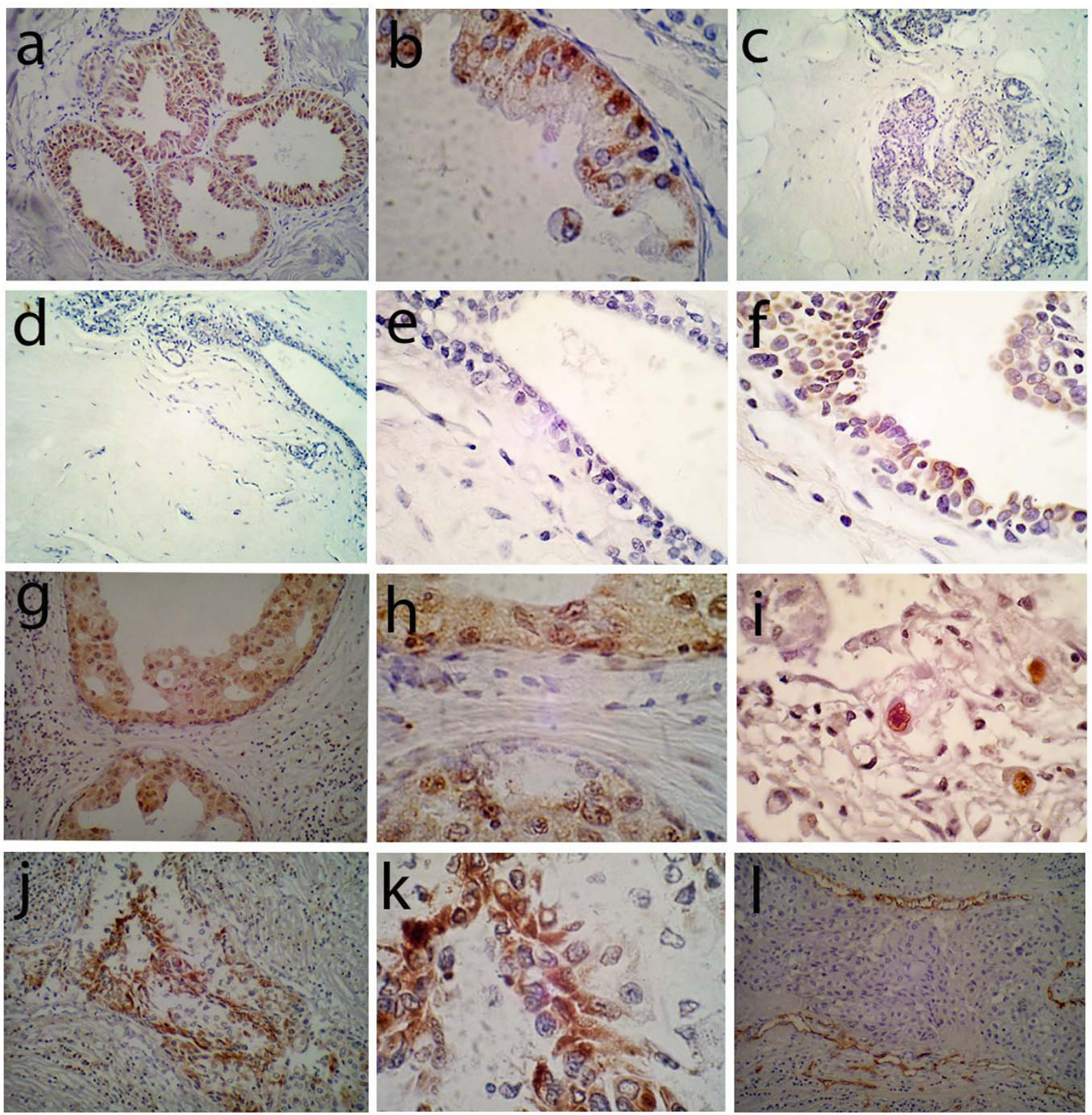

Figure 1 Immunohistochemical detection of HCMV in normal breast and breast cancer. (a, b) Examples of HCMV IE immuonreactivity in isolated area of normal ductal epithelium in normal breast from a reduction mammoplasty patient are presented. Low (a, 40x) and higher (b, 100x) power views of the same area of immunoreactive epithelium demonstrate discrete perinuclear and cytoplasmic epithelial cell staining. No IE immunoreactivity is detected in two different specimens obtained from normal reduction mammoplasty (c and d, 40x; e is 100x

magnification of d). IE immunoreactivity is demonstrated primarily in a nuclear distribution in matched non-neoplastic epithelium from a patient with infiltrative ductal carcinoma $(f, 100 x)$. Early and late $(E / L)$ immunoreactivity is demonstrated in the tumor epithelial cells, but not the stroma, from an area of ductal carcinoma in situ (DCIS) in a patient with infiltrative ductal carcinoma (g, 40x, h, 100x). Positive control immunostaining for IE immunoreactivity is shown in HCMV infected pneumocytes from an AIDS patient with CMV pneumonia (i, 100x). Late antigen (L) immunoreactivity is shown in another patient with infiltrative ductal carcinoma (j, 40x; k, 100x). Negative control (IE isotype control antibody staining for CD34) immunoreactivity is seen only in vascular endothelial cells in an area of infiltrative ductal carcinoma (I, 40x). 

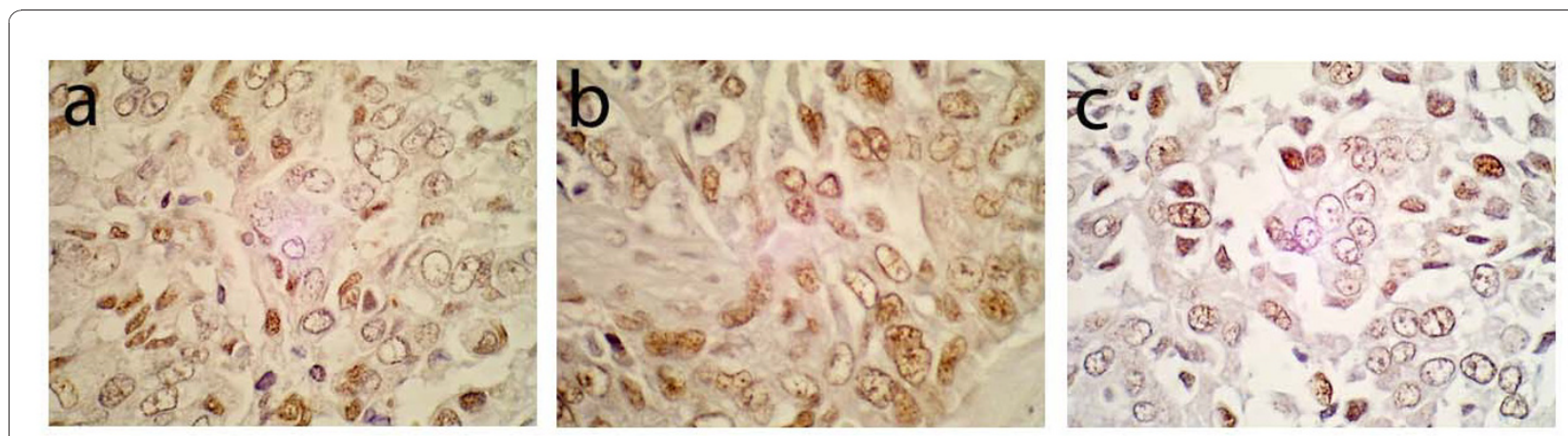

Figure 2 Immunohistochemical detection of three different HCMV antigens in a serial sections from infiltrative ductal carcinoma. Immunoreactivity with monoclonal antibodies specific for HCMV immediate early (IE; a), early and late $(E / L ; b)$, and late $(L ; C)$ antigens is shown in serial sections from a single specimen of infiltrative ductal carcinoma (100x).

Immunoreactivity was not detected in breast epithelium when primary antibody or monoclonal antibodies specific for smooth muscle actin and/or CD34 were used (Table 1), although immunoreactivity was evident in smooth muscle cells and endothelial cells with these antibodies as expected (e.g., Figure 1, l). The HCMV-IE, E/L and L monoclonal antibodies were specific for HCMV antigens when tested against known HCMV infected lung tissues from an AIDS patient (e.g, Figure 1, i).

Our analysis also demonstrated that there was a higher incidence of HCMV immunoreactivity in "tumor control" tissues from breast cancer patients than in "normal control" tissues from breast reduction mammoplasty (Table 2). Interestingly, while the incidence of HCMV IE immunoreactivity trended to a higher level in

Table 1 Comparisons of HCMV immunoreactivity in epithelium between breast cancer and non-neoplastic breast tissue from breast reduction mammoplasty

\begin{tabular}{lccccccr}
\hline & \multicolumn{2}{c}{ Tumor $(\mathbf{N}=\mathbf{3 9})$} & \multicolumn{4}{c}{$\begin{array}{c}\text { Normal Control } \\
(\mathbf{N}=\mathbf{3 8})\end{array}$} & $\begin{array}{r}\mathbf{p} \text { of Chi-Square } \\
\text { test }\end{array}$ \\
& $\begin{array}{c}\mathbf{N} \\
\text { (examined) }\end{array}$ & + & $\begin{array}{c}\mathbf{N} \\
\text { (examined) }\end{array}$ & + & \\
& & $\mathbf{n}$ & $\%$ & & $\mathbf{n}$ & $\%$ & 0.0009 \\
\hline $\mathrm{IE}$ & 32 & 31 & 97 & 27 & 17 & 63 & $<0.0001$ \\
$\mathrm{E} / \mathrm{L}$ & 25 & 21 & 84 & 28 & 6 & 21 & 0.2270 \\
$\mathrm{~L}$ & 27 & 15 & 56 & 28 & 11 & 39 & - \\
$\mathrm{No}$ & 19 & 0 & 0 & 20 & 0 & 0 & - \\
$\mathrm{Ab}$ & & & & & & & - \\
$\mathrm{CD} 34$ & 16 & 0 & 0 & 9 & 0 & 0 & - \\
Actin & 28 & 0 & 0 & 29 & 0 & 0 & - \\
\hline
\end{tabular}

Specific immunoreactivity to HCMV immediate early (IE) antigen and HCMV early and late $(E / L)$ antigens were significantly higher in breast cancer cases than normal controls. Immunoreactivity to the HCMV late (L) antigen was higher in breast cancer than normal breast. No immunoreactivity was detected in any specimen when primary antibody was omitted. All specimens had specific immunoreactivity to vascular endothelial cells and smooth muscle cells with anti-CD34 and anti-smooth muscle actin antibodies, respectively, but no immunostaining of epithelial cells was observed with these antibodies. the breast cancer patients' control tissues compared to the normal control tissues, it was not statistically significant. The incidence of HCMV E/L and L immunoreactivity was, however, significantly greater in the tumor control breast tissue compared to the normal control tissues (Table 2).

Of the tumor specimens and matched breast control specimens from tumor patients, a total of 30 specimens were analyzed with all three antibodies ( 25 tumor cases and 5 matched controls). 22 of the true normal breast tissues (from reduction mammoplasty) were also analyzed with all three antibodies. In all but one case where all three antibodies were tested in the tumor specimens, the specimens were positive for IE antibody staining (Table 1). In 3 of these cases both the $\mathrm{E} / \mathrm{L}$ and $\mathrm{L}$ antibody staining was negative, otherwise it was present. In 2 other cases either E/L or L antibody staining was

Table 2 Comparisons of HCMV immunoreactivity between nonneoplatic breast tissue from known cancer patients and non-neoplastic breast tissue from breast reduction mammoplasty normal conrols

\begin{tabular}{|c|c|c|c|c|c|c|c|}
\hline & \multicolumn{3}{|c|}{$\begin{array}{c}\text { Tumor Control } \\
(\mathrm{N}=21)\end{array}$} & \multicolumn{3}{|c|}{$\begin{array}{l}\text { Normal Control } \\
(\mathrm{N}=38)\end{array}$} & \multirow[t]{3}{*}{$\begin{array}{r}\mathrm{p} \text { of Chi-Square } \\
\text { test }\end{array}$} \\
\hline & \multirow{2}{*}{$\begin{array}{c}\mathrm{N} \\
\text { (examined) }\end{array}$} & \multicolumn{2}{|c|}{+} & \multirow{2}{*}{$\begin{array}{c}\mathrm{N} \\
\text { (examined) }\end{array}$} & \multicolumn{2}{|c|}{+} & \\
\hline & & $n$ & $\%$ & & $\mathbf{n}$ & $\%$ & \\
\hline$\overline{\mathrm{IE}}$ & 13 & 12 & 92 & 27 & 17 & 63 & $0.0678^{*}$ \\
\hline$E / L$ & 15 & 11 & 73 & 28 & 6 & 21 & 0.0009 \\
\hline $\mathrm{L}$ & 13 & 10 & 77 & 28 & 11 & 39 & 0.0249 \\
\hline $\begin{array}{l}\mathrm{No} \\
\mathrm{Ab}\end{array}$ & 9 & 0 & 0 & 20 & 0 & 0 & - \\
\hline CD34 & 6 & 0 & 0 & 9 & 0 & 0 & - \\
\hline Actin & 8 & 0 & 0 & 29 & 0 & 0 & - \\
\hline
\end{tabular}

* Two-sided Fisher's exact test

Specific immunoreactivity to HCMV E/L antigens was significantly higher in nonneoplastic breast from breast cancer cases than from breast reduction mammoplasty controls. Immunoreactivity to the HCMV late (L) antigen was also significantly higher in breast cancer than normal breast. 
negative while the other two antibodies were found to be positive.

In contrast, while 15 of the 22 true normal breast specimens from reduction mammoplasty patients were positive for IE antibody staining, only 7 of these 15 cases were also immunoreactive for $\mathrm{E} / \mathrm{L}$ and $\mathrm{L}$. These findings suggest that HCMV early and late antigen expression is less frequently found in normal breast tissues than in tumor tissues or normal appearing breast tissues from breast cancer patients.

To determine if the HCMV positivity rate of the tissue samples was due to the differences in the mean age or ethnicity of the control versus the cancer patient populations, we analyzed the data by assessing the HCMV IE1 immuonreactivity rate of patients $\leq 45$ years or $>45$ years of age. In $\leq 45$ years group, 7 of 8 (87.5\%) cancer patients had positive HCMV IE1 antigen, compared to 12 positives of 17 (70.6\%) normal tissue, and the difference was not significant. In $>45$ years group, all 20 cancer patients were positive for HCMV IE1 antigen, compared to 4 positives out of 7 normal tissues $(\mathrm{p}=$ 0.012). Thus, these data remained significant for patients $>45$ years of age.

There were 7 African-American patients and $31 \mathrm{Cau}-$ casian patients in the breast cancer group. There were 11 African-American and 21 Caucasian patients in the normal breast reduction mammoplasty group. For Caucasians, there was no significant difference between HCMV prevalence (95.7\% in cancer tissues compared to $88.9 \%$ in normal tissues). For African-Americans, HCMV prevalence was $100 \%$ in cancer tissues, while it was $50 \%$ in the normal tissues. However, this difference was not significant because of small numbers.

\section{In situ hybridization}

We performed in situ hybridization on breast cancer specimens and their paired control breast specimens, as well as normal controls from individuals with no history of breast cancer, to determine the presence of HCMV nucleic acids. We detected HCMV nucleic acids specifically in neoplastic or nonneoplastic epithelial cells in 16/ 18 randomly selected patients from the breast cancer pool. In $3 / 3$ cases that we tested both neoplastic and control epithelium from the same patient, HCMV nucleic acids were detected in both specimens. We also performed in situ hybridization on 18 randomly selected "normal control" breast specimens, and 11/18 of these specimens confirmed specific HCMV nucleic acid detection.

The pattern of HCMV immunoreactivity was similar to the pattern of ISH staining observed. We detected HCMV nucleic acid hybridization in normal breast epithelium and in neoplastic epithelium in areas of DCIS and IDC, but HCMV nucleic acids were principally undetectable in stromal cells (Figure 3, a-d). The pattern of HCMV nucleic acid detection within the cell was distinctly different from that of the Alu positive control probe, which is entirely specific to cellular nuclear DNA Alu repeats (Figure 3, e). In HCMV positive cells, we detected HCMV nucleic acids in the nucleus but also predominantly in the cell cytoplasm (as shown in Figure 3, b-d, and see Additional file 2). A positive control DNA probe (specific to DNA Alu repeat sequences) was specific for nuclear DNA in both epithelial and stromal cells in DCIS/IDC in tumors and normal breast (Figure 3, e), while a non-specific DNA probe was non-reactive in tumor and normal tissues (Figure 3, f). The specificity of HCMV nucleic acid hybridization was confirmed by detection of HCMV infected cells in HCMV infected lung tissues from an AIDS patient (Figure 3, g), while a negative control probe was negative in these same tissues (Figure 3, h). In normal breast epithelium from reduction mammoplasty patients, the amount of signal from HCMV nucleic acid hybridization was, in general, dramatically less than that found in breast tumor cells (e.g., Figure 3, i-j)

With respect to the internal consistency of immunohistochemical staining for HCMV antigens and detection of HCMV nucleic acids by in situ hybridization, there was a high correlation between positive for IHC and ISH specimens among the breast cancer and paired controls from breast cancer patients. 16/18 cases analyzed were positive for both HCMV antigen and nucleic acids, while in 2 cases there was a discordance (see Additional file 3).

In the normal breast tissue from non-cancer controls, there was a high degree of inconsistency between IHC and ISH results. Some of the positive IHC cases were negative for ISH, and vice versa (see Additional file 3). We attribute these results to the overall extremely low levels of antigen and nucleic acids detected in these normal tissues compared to the cancer cases. At such low levels of antigen and nucleic acid detection, we suspect that limitations of detection may have resulted in lack of internally consistent results between the two groups. In addition, it is possible that latent HCMV infection may occur in normal breast epithelium, in which case nucleic acids may be detected in the absence of protein expression.

\section{PCR and Sequencing}

We performed nested PCR for HCMV UL55 gene using DNA that was extracted from paraffin sections of 8 tumors and 4 control cases. $6 / 8$ tumor cases and $1 / 4$ normal control cases demonstrated amplified HCMV UL55 gene, which was confirmed by direct sequencing of the PCR products (not shown). Since the tumor specimens that were tested were all positive for HCMV by immunohistochemistry, we hypothesize that the negative PCR specimens may have had viral nucleic acids below the level of detection for our assay, or viral genetic 

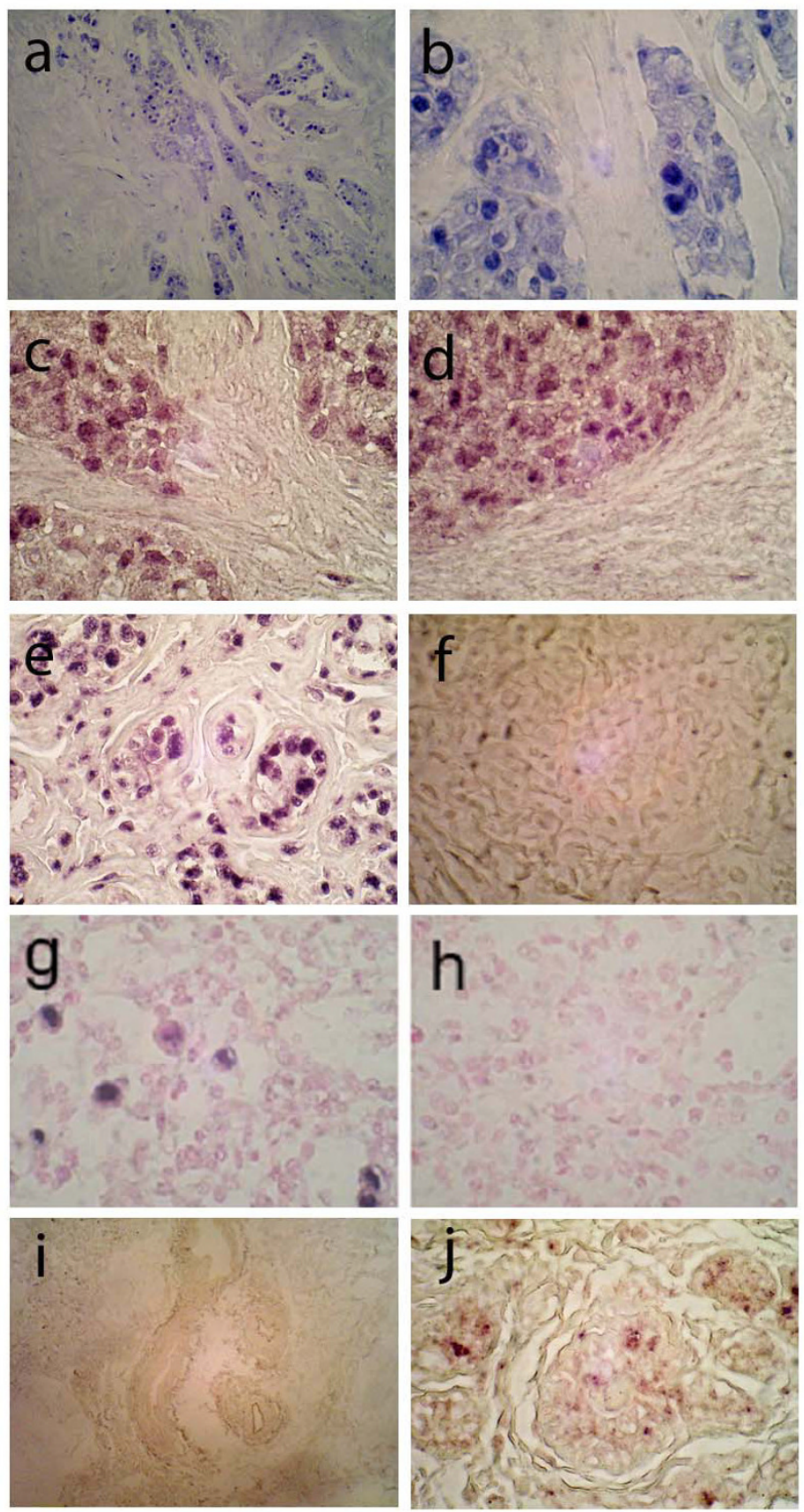

Figure 3 HCMV in situ hybridization in infiltrating ductal carcinoma. Two examples of HCMV ISH staining from two different patients with infiltrating ductal carcinoma are displayed. Low and high power images of the first patient $(\mathrm{a}, 40 \mathrm{x} ; \mathrm{b}, 100 \mathrm{x})$ demonstrate heterogeneous pattern of detection of HCMV nucleic acids in the nuclei (dark blue) and cytoplasm (light blue) in infiltrating tumor cells but no significant nucleic acid detection in the intervening stroma. Two images from another example of infiltrating ductal carcinoma (c, d; 100X) also reveal areas of HCMV nucleic acid detection in nuclear (dark purple) and cytoplasmic (light purple) areas, without any significant nucleic acid detection in the intervening stroma. Positive control probe specific for DNA Alu repeats from the same case reveals intense (dark purple) nuclear probe hybridization with nucleic acids in infiltrating tumor nuclei and intervening stromal cells, without evidence of cytoplasmic staining (e, 100x). Hybridization signal of a negative control probe specific to insect nucleic acids is completely absent in tumor tissues (f, 100x). Positive control (HCMV infected lung) is positive for HCMV nucleic acid hybridization in scattered pneumocytes (blue cells in g, 100x), while negative control probe is not detected in the same specimens ( $h, 100 x$; light hematoxylin counterstain was used in $g$ and $h$ ). Low power image (i, 40x) of normal breast epithelium (from reduction mammoplasty) that was negative for HCMV nucleic acid hybridization reveals no hybridization signal. High power $(j, 100 x)$ image of normal breast epithelium from reduction mammoplasty reveals faint purple hybridization signal in scattered normal ductal epithelial cells. 
material may have been more degraded than cellular housekeeping genes used as controls, since the DNA quality in these assays is very variable. Alternatively, the paraffin specimens used for these assays, which were not necessarily sequential sections to the ones used for immunohistochemistry and in situ hybridization, may have had less viral genome present. The one case of normal breast control tissue that was positive for HCMV UL55 by PCR was also positive for HCMV by immunohistochemistry and in situ hybridization, while the remaining three cases were negative by all three detection methods.

\section{Discussion}

Breastfeeding is the major route of HCMV transmission during the first year of life in countries where most women are seropositive and breast-feed their infants [36]. Since cell free virus is shed in the breast milk in virtually all HCMV seropositive females, the natural reservoir for HCMV in the breast is likely breast glandular epithelial cells. We demonstrate here that 17/27 of the normal breast specimens in our study from females with no history of breast cancer exhibited evidence of persistent HCMV infection as determined by HCMV-IE antigen expression.

Unexpectedly, we found that 31/32 (97\%) of cases of breast carcinoma in our study also have evidence of HCMV infection and expression based upon immunohistochemistry. Immunoreactivity to non-IE HCMV antigens was detected in a significantly higher percentage of breast cancer specimens than normal breast cases. Overall, these data indicate that persistent HCMV infection occurs specifically in breast glandular epithelium for a significant percentage of normal adult females and that HCMV IE protein expression is significantly associated with neoplastic compared to nonneoplastic breast glandular epithelium in patients over age 45 in our group.

Our data are consistent with a previous PCR-based report that indirectly suggested HCMV infection is present in breast cancer [40]. In this study, the investigators analyzed 12 specimens of normal breast from a noncancer group, and 62 samples of invasive ductal carcinoma from breast cancer patients for several DNA viruses using DNA PCR followed by Southern hybridization [40]. The viruses analyzed included human papillomavirus (HPV), HCMV, EBV, herpes simplex virus 1 (HSV-1), HSV-2, and human herpesvirus - 8 (HHV-8). Of these six DNA viruses, only HCMV was detected in normal breast $(8 / 12 ; 67 \%)$ specimens. HCMV DNA was also detected in $47 / 62$ (76\%) of invasive ductal carcinoma specimens. Since in situ techniques were not used in this study, no clear conclusion that HCMV was located in tumor epithelial cells could be made.
Our novel findings of the expression of the IE antigen and other gene products in normal and neoplastic breast epithelial cells indicate that the breast epithelium is a reservoir for persistent HCMV infection. While this phenomenon has not been previously demonstrated, it is not completely unexpected. HCMV is known to be able to infect multiple organs, including the salivary glands, lung, gastrointestinal tract, kidney, liver, spleen and brain [41-43]. The best candidate cells for latent infection are thought to be monocytes [44]. However, chronic infection and expression of HCMV gene products in normal breast epithelium may represent a critical component of the viral life-cycle, since breast milk is a major mode of transmission and survival for the virus.

A well known consequence of persistent viral infection and inflammation is neoplastic transformation. Indeed, an increasing percentage of human malignancies in the last several decades have been attributed to chronic infection and chronic inflammation [45]. It is well established that chronic inflammation plays a critical role in the transition from neoplastic precursor to full-blown invasive malignancy, and inflammation is considered the seventh hallmark of neoplasia [46-48]. This period of chronic inflammation may indeed be essential for the neoplastic process in malignancy, and may be facilitated by infectious agents that act as "promoters". For example, Hepatitis $C$ virus chronically infects the liver and causes a persistent inflammatory immune response resulting in hepatoma[49]. Another example is Epstein Barr virus (EBV) in nasopharyngeal carcinoma. EBV is ubiquitous in the human population, and thus is not oncogenic under normal circumstances. However, EBV infection in the nasopharynx of individuals exposed to certain environmental carcinogens is critical in the development of nasopharyngeal carcinoma, through expression of latent EBV genes that promote cell growth and survival [50].

An accumulating body of evidence indicates that HCMV gene expression in normal epithelial cells, tumor cells and tumor infiltrating macrophages could promote an oncogenic environment. Multiple HCMV gene products are known to promote mutagenesis and to dysregulate cell cycle checkpoint controls, and drive oncogenic signaling pathways (reviewed in [26]). Recent experimental evidence has shown that the chronic expression of TNF-alpha and IL-1 beta in the pre-malignant microenvironment in the setting of inflammation can produce dramatic increases in the likelihood of malignant transformation via activation of the NF-kB transcriptional activator [51]. Furthermore, two critical downstream effectors of this NF-kB pathway with respect to oncogenicity appear to be COX-2 and IL-6 $[49,52,53]$. IL-6 induction and expression in tumor cells and tumor associated myeloid cells has an important 
role in chronic inflammatory oncogenic signaling, likely by activation of the STAT-3 transcriptional activator [54]. Hence, the NF-kB pathway has a dual effect in tumor promotion by preventing cell death of cells with malignant potential and by stimulating pro-inflammatory cytokines in infiltrating myeloid and lymphoid cells. Chronic HCMV infection could potentially promote these important oncogenic signaling pathways since HCMV infection expresses a chemokine receptor US28, which has oncogenic potential and has been shown to signal through the NF-kB pathway and activate downstream COX-2, STAT-3 and IL-6 expression $[55,56]$. Indeed, an etiological role for HCMV in breast cancer has been hypothesized based on epidemiological considerations, and investigators have demonstrated that breast cancer patients have increased IgG antibody titers to HCMV compared to controls $[57,58]$.

\section{Conclusion}

The data presented here indicate that HCMV infection occurs in normal breast epithelium in a majority of adult females evaluated and that a high percentage of breast cancer specimens have evidence of HCMV infection. These findings suggest that further research in this area is warranted to determine whether HCMV infection of breast epithelium represents an important factor in the initiation and promotion of breast cancer, and raise the possibility that in the future, antiviral based strategies may play a role in the management of this disease.

\section{Additional material}

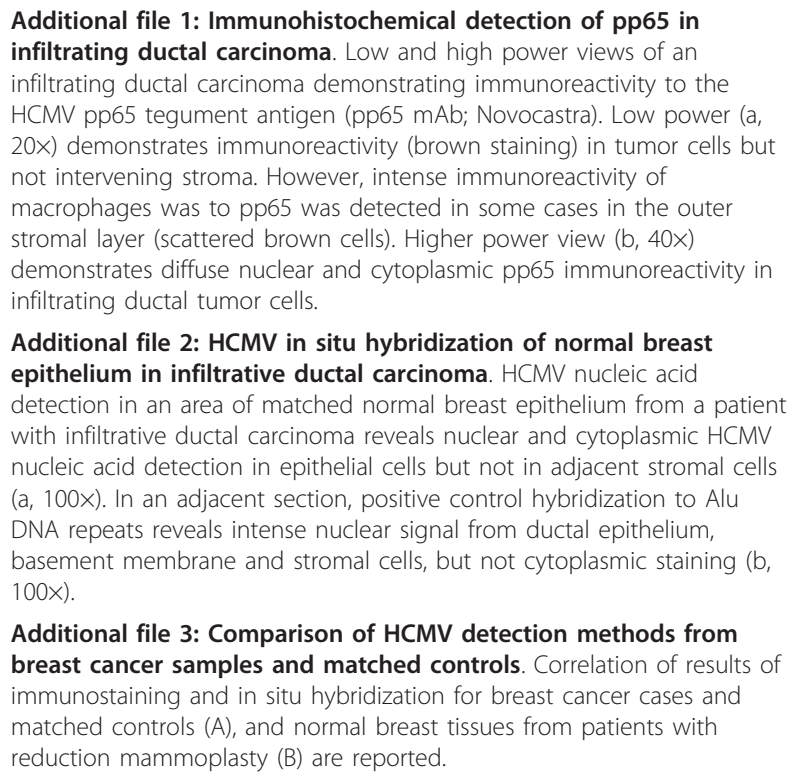

Additional file 3: Comparison of HCMV detection methods from breast cancer samples and matched controls. Correlation of results of immunostaining and in situ hybridization for breast cancer cases and matched controls (A), and normal breast tissues from patients with reduction mammoplasty (B) are reported.

\section{List of Abbreviations}

DCIS: ductal carcinoma in situ; EBV: Epstein Barr Virus; IDC: infiltrating ductal carcinoma; IE: Immediate early; IHC: immunohistochemistry; ISH: in situ hybridization; HCMV: human cytomegalovirus; L, late; IE/L, immediate early and late.

\section{Acknowledgements}

The authors wish to thank Dr. Andea Volk for assisting in preliminary pathological studies. This work was supported with pilot funds from the UAB Breast Cancer NCI SPORE project.

\section{Author details}

${ }^{1}$ Department of Pathology and Laboratory Medicine, Birmingham Veterans Hospital, 700 South 19th Street, Birmingham, AL, 35233, USA. ${ }^{2}$ Research Institute, California Pacific Medical Center, (475 Brannan St., Suite 220) San Francisco CA, 94107, USA. ${ }^{3}$ Pediatrics, University of Alabama at Birmingham, (1600 7th Avenue South), Birmingham AL, 35233 USA. ${ }^{4}$ UAB Comprehensive Cancer Center Biostatistics Facility, University of Alabama at Birmingham, (1802 6th Avenue South), Birmingham AL, 35233 USA. ${ }^{5}$ Surgery, University of Alabama at Birmingham, (1802 6th Avenue South), Birmingham AL, 35233 USA.

\section{Authors' contributions}

LH performed the immunohistochemistry and in situ hybridization studies. LM assisted in writing and preparing data for the study. KK scored slides for immunoreactivity. WW participated in the design of the study and performed the statistical analysis. WB, LS and KB helped in the study design and drafting of the manuscript. CC conceived of the study, and participated in its design and coordination, analyzed the data and drafted the manuscript. All authors have read and approved the final manuscript.

\section{Competing interests}

The authors declare that they have no competing interests.

Received: 9 August 2010 Accepted: 23 December 2010

Published: 23 December 2010

\section{References}

1. Britt WJAC: Fields Virology. In Cytomegalovirus. Third edition. Edited by: KD Fields BN, Howley PM. New York: Raven Press; 1996.

2. McPherson $K$, Steel CM, Dixon JM: $A B C$ of breast diseases. Breast cancerepidemiology, risk factors, and genetics. BMJ 2000, 321(7261):624-8.

3. Mant C, et al: A viral aetiology for breast cancer: time to re-examine the postulate. Intervirology 2004, 47(1):2-13.

4. Preciado MV: Lack of evidence for an association of Epstein-Barr virus infection with breast carcinoma-another point of view. Breast Cancer Res 2003, 5(4):E6, author reply E7.

5. Herrmann K, Niedobitek G: Lack of evidence for an association of EpsteinBarr virus infection with breast carcinoma. Breast Cancer Res 2003, 5(1):R13-7.

6. Labrecque LG, et al: Epstein-Barr virus in epithelial cell tumors: a breast cancer study. Cancer Res 1995, 55(1):39-45.

7. Xue SA, et al: Epstein-Barr virus gene expression in human breast cancer: protagonist or passenger? Br J Cancer 2003, 89(1):113-9.

8. Wang $Y$, et al: High prevalence of MMTV-like env gene sequences in gestational breast cancer. Med Oncol 2003, 20(3):233-6.

9. Pogo BG, et al: Sequences homologous to the mouse mammary tumor virus env gene in human breast carcinoma correlate with overexpression of laminin receptor. Clin Cancer Res 1999, 5(8):2108-11.

10. Ford CE, Faedo M, Rawlinson WD: Mouse mammary tumor virus-like RNA transcripts and DNA are found in affected cells of human breast cancer. Clin Cancer Res 2004, 10(21):7284-9.

11. Melana SM, et al: Characterization of viral particles isolated from primary cultures of human breast cancer cells. Cancer Res 2007, 67(18):8960-5.

12. Katz $E$, et al: MMTV Env encodes an ITAM responsible for transformation of mammary epithelial cells in three-dimensional culture. J Exp Med 2005, 201(3):431-9.

13. Ross SR, et al: An immunoreceptor tyrosine activation motif in the mouse mammary tumor virus envelope protein plays a role in virus-induced mammary tumors. J Virol 2006, 80(18):9000-8. 
14. Gumus M, et al: HPV DNA frequency and subset analysis in human breast cancer patients' normal and tumoral tissue samples. J Exp Clin Cancer Res 2006, 25(4):515-21.

15. Heng $B$, et al: Human papilloma virus is associated with breast cancer. $\mathrm{Br}$ J Cancer 2009, 101(8):1345-50.

16. Lawson JS, et al: Koilocytes indicate a role for human papilloma virus in breast cancer. Br J Cancer 2009, 101(8):1351-6.

17. Lawson JS, et al: Mouse mammary tumor virus-like sequences in human breast cancer. Cancer Res 2010, 70(9):3576-85.

18. Dimri $G$, Band $H$, Band V: Mammary epithelial cell transformation: insights from cell culture and mouse models. Breast Cancer Res 2005, 7(4):171-9.

19. Mitchell DA, et al: Sensitive detection of human cytomegalovirus in tumors and peripheral blood of patients diagnosed with glioblastoma. Neuro Oncol 2008, 10(1):10-8.

20. Zafiropoulos A, et al: Human herpes viruses in non-melanoma skin cancers. Cancer Lett 2003, 198(1):77-81.

21. Samanta M, et al: High prevalence of human cytomegalovirus in prostatic intraepithelial neoplasia and prostatic carcinoma. J Urol 2003, 170(3):998-1002

22. Harkins $L$, et al: Specific localisation of human cytomegalovirus nucleic acids and proteins in human colorectal cancer. Lancet 2002, 360(9345):1557-63

23. Castillo JP, Yurochko AD, Kowalik Tf: Role of human cytomegalovirus immediate-early proteins in cell growth control. J Virol 2000, 74(17):8028-37.

24. Cinatl J Jr, et al: Oncomodulatory signals by regulatory proteins encoded by human cytomegalovirus: a novel role for viral infection in tumor progression. FEMS Microbiol Rev 2004, 28(1):59-77.

25. Cobbs CS, et al: Human cytomegalovirus infection and expression in human malignant glioma. Cancer Res 2002, 62(12):3347-50.

26. Michaelis M, Doerr HW, Cinatl J: The story of human cytomegalovirus and cancer: increasing evidence and open questions. Neoplasia 2009, 11(1):1-9.

27. Soderberg-Naucler C: Does cytomegalovirus play a causative role in the development of various inflammatory diseases and cancer? J Intern Med 2006, 259(3):219-46.

28. Bego MG, Jeor SSt: Human cytomegalovirus infection of cells of hematopoietic origin: HCMV-induced immunosuppression, immune evasion, and latency. Exp Hematol 2006, 34(5):555-70.

29. Kalejta RF, Shenk T: Manipulation of the cell cycle by human cytomegalovirus. Front Biosci 2002, 7:d295-306.

30. Shen $Y$, Zhu H, Shenk T: Human cytomagalovirus IE1 and IE2 proteins are mutagenic and mediate "hit-and-run" oncogenic transformation in cooperation with the adenovirus E1A proteins. Proc Natl Acad Sci USA 1997, 94(7):3341-5.

31. Zhu H, Shen Y, Shenk T: Human cytomegalovirus IE1 and IE2 proteins block apoptosis. J Virol 1995, 69(12):7960-70.

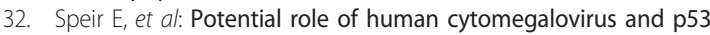
interaction in coronary restenosis. Science 1994, 265(5170):391-4.

33. Basta S, Bennink JR: A survival game of hide and seek: cytomegaloviruses and MHC class I antigen presentation pathways. Viral Immunol 2003, 16(3):231-42.

34. Asanuma $\mathrm{H}$, et al: Role of milk whey in the transmission of human cytomegalovirus infection by breast milk. Microbiol Immunol 1996, 40(3):201-4.

35. Hamprecht $\mathrm{K}$, et al: Epidemiology of transmission of cytomegalovirus from mother to preterm infant by breastfeeding. Lancet 2001, 357(9255):513-8.

36. Stagno S, Cloud GA: Working parents: the impact of day care and breastfeeding on cytomegalovirus infections in offspring. Proc Natl Acad Sci USA 1994, 91(7):2384-9.

37. Hotsubo T, et al: Detection of human cytomegalovirus DNA in breast milk by means of polymerase chain reaction. Microbiol Immunol 1994, 38(10):809-11.

38. Hamprecht $K$, et al: Detection of cytomegaloviral DNA in human milk cells and cell free milk whey by nested PCR. J Virol Methods 1998, 70(2):167-76.

39. Kuhn JE, et al: Quantitation of human cytomegalovirus genomes in the brain of AIDS patients. J Med Virol 1995, 47(1):70-82
40. Tsai $\mathrm{JH}$, et al: Association of viral factors with non-familial breast cancer in Taiwan by comparison with non-cancerous, fibroadenoma, and thyroid tumor tissues. J Med Virol 2005, 75(2):276-81.

41. Hahn G, Jores R, Mocarski ES: Cytomegalovirus remains latent in a common precursor of dendritic and myeloid cells. Proc Natl Acad Sci USA 1998, 95(7):3937-42.

42. Hendrix $M G$, et al: High prevalence of latently present cytomegalovirus in arterial walls of patients suffering from grade III atherosclerosis. Am J Pathol 1990, 136(1):23-8.

43. Hendrix RM, et al: Widespread presence of cytomegalovirus DNA in tissues of healthy trauma victims. J Clin Pathol 1997, 50(1):59-63.

44. Boppana SB, Britt WJ: Recognition of human cytomegalovirus gene products by HCMV-specific cytotoxic T cells. Virology 1996, 222(1):293-6.

45. Demaria $S$, et al: Cancer and inflammation: promise for biologic therapy. $\mathrm{J}$ Immunother 33(4):335-51.

46. Colotta F, et al: Cancer-related inflammation, the seventh hallmark of cancer: links to genetic instability. Carcinogenesis 2009, 30(7):1073-81.

47. Balkwill F, Charles KA, Mantovani A: Smoldering and polarized inflammation in the initiation and promotion of malignant disease. Cancer Cell 2005, 7(3):211-7.

48. Grivennikov SI, Greten FR, Karin M: Immunity, inflammation, and cancer. Cell 140(6):883-99.

49. Berasain $C$, et al: Inflammation and liver cancer: new molecular links. Ann N Y Acad Sci 2009, 1155:206-21.

50. Young LS, Rickinson AB: Epstein-Barr virus: 40 years on. Nat Rev Cancer 2004, 4(10):757-68.

51. Greten FR, et al: IKKbeta links inflammation and tumorigenesis in a mouse model of colitis-associated cancer. Cell 2004, 118(3):285-96.

52. Karin M: NF-kappaB as a critical link between inflammation and cancer. Cold Spring Harb Perspect Biol 2009, 1(5):a000141.

53. Fantini MC, Pallone F: Cytokines: from gut inflammation to colorectal cancer. Curr Drug Targets 2008, 9(5):375-80.

54. Tanabe $K$, et al: Mechanisms of tumor necrosis factor-alpha-induced interleukin-6 synthesis in glioma cells. J Neuroinflammation 7:16.

55. Maussang D, et al: The human cytomegalovirus-encoded chemokine receptor US28 promotes angiogenesis and tumor formation via cyclooxygenase-2. Cancer Res 2009, 69(7):2861-9.

56. Slinger $E_{\text {, et }}$ al: HCMV-encoded chemokine receptor US28 mediates proliferative signaling through the IL-6-STAT3 axis. Sci Signal 3(133):ra58

57. Richardson A: Is breast cancer caused by late exposure to a common virus? Med Hypotheses 1997, 48(6):491-7

58. Richardson AK, et al: Cytomegalovirus, Epstein-Barr virus and risk of breast cancer before age 40 years: a case-control study. Br J Cancer 2004, 90(11):2149-52.

doi:10.1186/2042-4280-1-8

Cite this article as: Harkins et al:: Detection of human cytomegalovirus in normal and neoplastic breast epithelium. Herpesviridae 2010 1:8.

\section{Submit your next manuscript to BioMed Central and take full advantage of:}

- Convenient online submission

- Thorough peer review

- No space constraints or color figure charges

- Immediate publication on acceptance

- Inclusion in PubMed, CAS, Scopus and Google Scholar

- Research which is freely available for redistribution

Submit your manuscript at www.biomedcentral.com/submit
C Biomed Central 